\title{
Trigeminal Neuralgia Associated with the Specific Bridging Pattern of Transverse Pontine Vein: Diagnostic Value of Three-Dimensional Multifusion Volumetric Imaging
}

\author{
Makoto Oishi ${ }^{a} \quad$ Masafumi Fukuda ${ }^{a}$ Yoshiyuki Noto ${ }^{b}$ Tadashi Kawaguchic $^{c}$ \\ Tetsuya Hiraishia Yukihiko Fujii ${ }^{a}$ \\ ${ }^{a}$ Department of Neurosurgery, Brain Research Institute, Niigata University, ${ }^{b}$ Department of Radiology, \\ Niigata University Hospital, and ' Department of Neurosurgery, Nagaoka Red Cross Hospital, Niigata, Japan
}

\section{Key Words}

Computed tomography $\cdot$ Magnetic resonance imaging •

Microvascular decompression · 3-Dimensional imaging •

Transverse pontine vein · Trigeminal neuralgia

\begin{abstract}
Objectives: We report the specific bridging pattern of a transverse pontine vein (TPV) associated with trigeminal neuralgia (TN), which was evaluated by 3-dimensional (3D) multifusion volumetric imaging (MFVI). Methods: In 3 cases with TN (V1 or V1-2 territory), constructive interference in steady state (CISS) imaging confirmed no arterial compression but indicated a vein draining into Meckel's cave. Virtual endoscopic (VE) analysis for CISS images and 3D MFVI (in 2 cases) including venous information was obtained by a multidetector row computed tomography (MDCT) system. Additionally, we investigated the bridging pattern of veins around Meckel's cave on 3D MFVI of 50 cerebellopontine angle (CPA) regions without any lesions. Results: In all 3 patients, VE of CISS or 3D MFVI identified a bridging vein from the TPV causing the focal deformity of the trigeminal nerve near Meckel's cave. All those patients achieved a pain-free state after surgically coagulating and cutting the vein. In in-
\end{abstract}

vestigating 3D MFVI of 50 CPA regions, this type of the bridging vein was found in 4 (8\%) including the presented 2 cases. Conclusions: The specific bridging pattern of the TPV draining into Meckel's cave can be associated with TN. The 3D MFVI analysis using venous information obtained by MDCT was useful to evaluate surgical anatomy including the offending vein which can be missed.

Copyright $\odot 2011$ S. Karger AG, Basel

\section{Introduction}

Trigeminal neuralgia (TN) is a hyperactive dysfunction syndrome of the trigeminal nerve, and its mechanism has been explained as vascular compression to the nerve and consecutive demyelination [1]. Microvascular decompression (MVD) surgery, which was first reported in 1967 [2], has been widely accepted as the curative treatment for $\mathrm{TN}$. The main vessel causing $\mathrm{TN}$ is the superior cerebellar artery (SCA), which is confirmed in $75-80 \%$ of all patients [3-5], and the postsurgical curative rate after MVD for this arterial compression is $70-80 \%$ among the long-term outcomes $[3,5]$. Venous compression alone as the cause of $\mathrm{TN}$ is observed at a relatively lower rate, i.e.

\section{KARGER}

Fax +4161306 1234 E-Mail karger@karger.ch www.karger.com
(C) 2011 S. Karger AG, Basel

1011-6125/11/0894-0226\$38.00/0

Accessible online at:

www.karger.com/sfn
Makoto Oishi, MD

Department of Neurosurgery, Brain Research Institute, Niigata University

1-757 Asahimachidori, Chuo-Ku

Niigata 951-8585 (Japan)

Tel. +81252270653,E-Mail mac.oishi@mac.com 
$6-18 \%[3,4,6-8]$. Only a few authors have reported some differences of its clinical characteristics and surgical outcomes in comparison with arterial compressions [8-10].

The advent of the high resolution of magnetic resonance imaging (MRI) has undoubtedly contributed to improvement of the clinical ability to diagnose neurovascular compression (NVC) syndromes including TN. Application of the recent 3-dimensional (3D) image-analyzing techniques has enabled a precise understanding of the anatomical relationship of structures in the cerebellopontine angle (CPA) region, which was difficult to understand from 2-dimensional (2D) images [11-16]. Recently, $3 \mathrm{D}$ computed tomographic angiography (CTA) with a multidetector row CT (MDCT) system has been used to demonstrate the $3 \mathrm{D}$ vascular information of the arterial and venous phase separately $[17,18]$. The resolution of venous information provided by this method is sufficient to evaluate relatively small veins bridging in the CPA cistern.

In the present article, we report our experiences of 3 surgical cases with TN caused only by a vein presenting the similar bridging pattern. Herein, we present the specific utility of 3D multifusion volumetric imaging (MFVI), which was characterized by 'volume-rendering' and 'image fusion' techniques for radiological data $[15,16,19]$ and in which venous information obtained from CTA was integrated, for the diagnosis and presurgical simulation of the patients. Additionally, we investigated the bridging patterns of the presented vein by evaluating the $50 \mathrm{CPA}$ regions of patients with NVC on 3D MFVI.

\section{Patients and Methods}

\section{Patients}

Between 2005 and 2009, we used MVD surgery for 35 patients with TN. In the following 3 patients, only a vein was identified as the offending vessel.

Case 1. A 68-year-old woman presented with an almost 4-year history of right-sided facial pain, which became uncontrollable after treatment with carbamazepine. She experienced pain in the V1 territory, which was often triggered by cold air and sometimes affected the V2 territory.

Case 2. A 30-year-old woman visited our hospital with medically intractable right-sided facial pain limited to the V1 territory. She had undergone MVD surgery at another hospital 1 year previously, but her pain was not reduced postoperatively.

Case 3. A 62-year-old woman developed right-sided facial pain for 6 years. We performed MVD of the SCA due to the diagnosis of arterial compression of the trigeminal nerve. She felt the same pain just after surgery but it disappeared within a week. The same pain recurred 1 year later and worsened with time. Her pain was limited mainly to the V2 territory, but sometimes spread to the V1 territory. She was administered toxic doses of carbamazepine.

\section{Imaging Data Acquisition}

MRI scanning was performed on a 1.5 -tesla MRI system (Magnetom Symphony; Siemens AG, Munich, Germany). MR cisternography was performed with a $3 \mathrm{D}$ constructive interference in steady state (CISS) sequence with the following parameters: TR, $10.15 \mathrm{~ms}$; TE, $4.48 \mathrm{~ms}$; flip angle, $70^{\circ}$; field of view, $200 \mathrm{~mm}$; matrix, $512 \times 384$; slice thickness, $0.80 \mathrm{~mm}$; 1 acquisition.

CT scanning was performed using a 64-section MDCT system (Aquilon; Toshiba, Tokyo, Japan); the slice thickness was $0.3 \mathrm{~mm}$, and the scan speed was $0.75 \mathrm{~s} /$ cycle. For scanning CTA, the patient was administered a venous injection of iodized contrast medium (maximum injection volume, $95 \mathrm{ml}$ ), and, in total, 3 scanning sessions were performed - before injection, immediately after injection and some time after injection, to separately obtain the skull, arterial and venous data.

\section{Creating 3D MFVI Data}

We created 3D MFVI data by using a commercially available image analysis software (Real INTAGE; Cybernet Inc., Tokyo, Japan), which enabled the integration of multiple data obtained using different modalities by an 'image fusion' technique and reconstruction of the volumetric data from the sequential slice images by employing a 'volume-rendering' technique [20]. The details of manipulating the software were described elsewhere $[15,16,19]$. Briefly, the coordinates of all the data sets were manually adjusted with special attention on the margins of cerebrospinal fluid (CSF) signals, bone density and vascular information. Subsequently, the volumetric data of the skull, arteries and veins extracted from the CT and CTA data were integrated into the volumetric data of CISS.

\section{Reviewing the 3D MFVI Data and Virtual Endoscopic} Analysis

The 'fly-through' algorithm can create the dynamic $3 \mathrm{D}$ views in the volumetric data from a 'virtual camera', which can be moved and rotated in a complex way [20]. This function has already been introduced as virtual endoscopy (VE) $[15,16]$. The viewing angle was fixed at the usual $80^{\circ}$. The position and the projection of the virtual camera were indicated on multiplanar reconstruction 2D images. We created 3D MFVI data to obtain the VE views of fine anatomy in the cistern by adjusting the window level, window width and opacity to cause the CSF signals to be transparent while keeping the signals of structures.

\section{Additional Evaluation of the Venous Anatomy around the \\ Trigeminal Nerve}

We performed an additional investigation about the bridging patterns of the vein around the trigeminal nerve. We analyzed 3D MFVI data of 50 sides in 25 patients with hemifacial spasm or TN. We selected those cases as materials to be regarded as presenting a nearly normal anatomy in terms of venous drainage because of no tumor or vascular lesion. All the patients had undergone MVD between February 2008 and September 2009. Cases 2 and 3 were included in the materials but case 1 was not included because the present protocol of CTA scanning was not applied yet. 

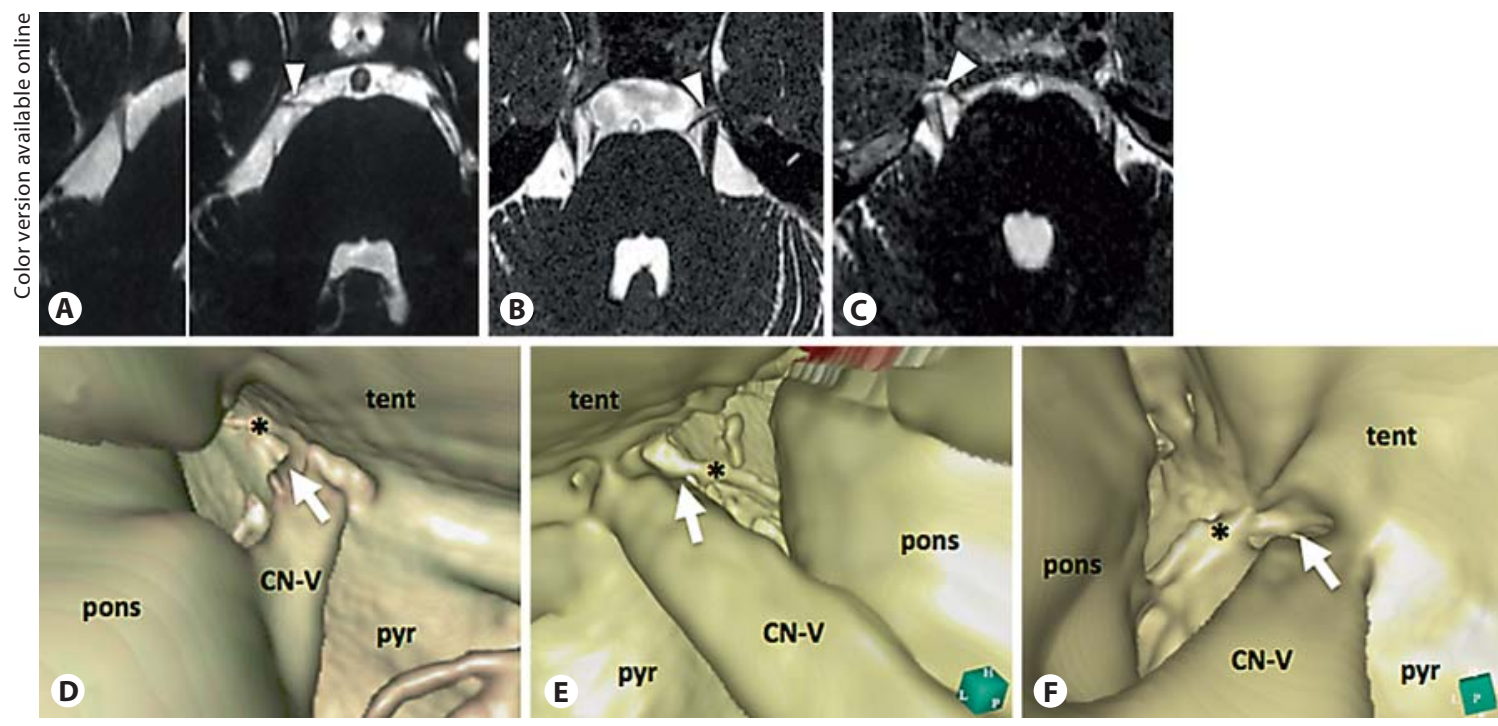

Fig. 1. A-C The trigeminal nerve without any arterial compression and a linear structure bridging into Meckel's cave from the surface of the brainstem (arrowheads) are clearly observed on the 2D images of CISS in cases 1 (A), 2 (B) and 3 (C). D-F The VE views of CISS data in cases 1 (D), 2 (E) and 3 (F) visualize a trigeminal nerve with the compressed deformity (arrows) at the superomedial site by the linear structure $\left(^{*}\right)$ near the entrance of Meckel's cave. $\mathrm{CN}-\mathrm{V}=$ Trigeminal nerve; pyr = pyramis; tent = tentorium.

Table 1. Clinical and surgical results of 3 cases

\begin{tabular}{|c|c|c|c|c|c|c|}
\hline Case & Age/sex & $\begin{array}{l}\text { Territory } \\
\text { of pain }\end{array}$ & $\begin{array}{l}\text { Approach/procedure for } \\
\text { MVD }\end{array}$ & Postsurgical course & Final outcome & Complication \\
\hline 1 & 68 years $/ F$ & right V1 & $\begin{array}{l}\text { retrosigmoid/coagulation } \\
\text { and cutting of the vein }\end{array}$ & $\begin{array}{l}\text { complete disappearance } \\
\text { immediately after surgery }\end{array}$ & $\begin{array}{l}\text { pain free for } \\
46 \text { months }\end{array}$ & $\begin{array}{l}\text { hypesthesia } \\
50 \%(\mathrm{~V} 1-2)\end{array}$ \\
\hline 2 & 30 years/F & left V1 & $\begin{array}{l}\text { retrosigmoid/coagulation } \\
\text { and cutting of the vein }\end{array}$ & $\begin{array}{l}\text { complete disappearance } \\
\text { at } 3 \text { months after surgery }\end{array}$ & $\begin{array}{l}\text { pain free for } \\
24 \text { months }\end{array}$ & none \\
\hline 3 & 62 years $/ F$ & $\begin{array}{l}\text { right V1, V2 } \\
(\mathrm{V} 2>\mathrm{V} 1)\end{array}$ & $\begin{array}{l}\text { retrosigmoid/coagulation } \\
\text { and cutting of the vein }\end{array}$ & $\begin{array}{l}\text { complete disappearance } \\
\text { immediately after surgery }\end{array}$ & $\begin{array}{l}\text { pain free for } \\
18 \text { months }\end{array}$ & none \\
\hline
\end{tabular}

\section{Results}

A summary of clinical characteristics, 3D MFVI and surgical results of all the 3 cases are shown in table 1 .

Findings around the Trigeminal Nerve on $3 D$ MFVI

A review of the 2D images of CISS showed the linear structure indicating the bridging vein from the transverse pontine vein (TPV) running into Meckel's cave in all patients, but the compression to the trigeminal nerve by this vein was inconclusive (fig. $1 \mathrm{~A}-\mathrm{C}$ ). VE analysis of
3D CISS indicated that all the linear structures continued into Meckel's cave and made a focal deformity on the nerve, such as a dent (fig. 1D-F).

A review of 3D MFVI data allowed visualization of anatomies around the trigeminal nerve with sufficient quality microsurgical resolution. In case 1 , because the present 3D CTA protocol was not applied yet, 3D MFVI data were created only from CISS and MR angiography (fig. 2). In cases 2 and 3, 3D MFVI was created by integration of CISS and CTA data (fig. 3). The linear structure was confirmed to definitely be a vein, which should cor- 
Fig. 2. The presurgical VE view (A) was compatible with the actual operative view (B) in case 1 with right TN. The linear structure $\left({ }^{*}\right)$ was confirmed as the vein bridging into Meckel's (M's) cave, and the nerve compression by the vein was also similarly observed on both views. $\mathrm{CN}-\mathrm{V}=$ Trigeminal nerve.
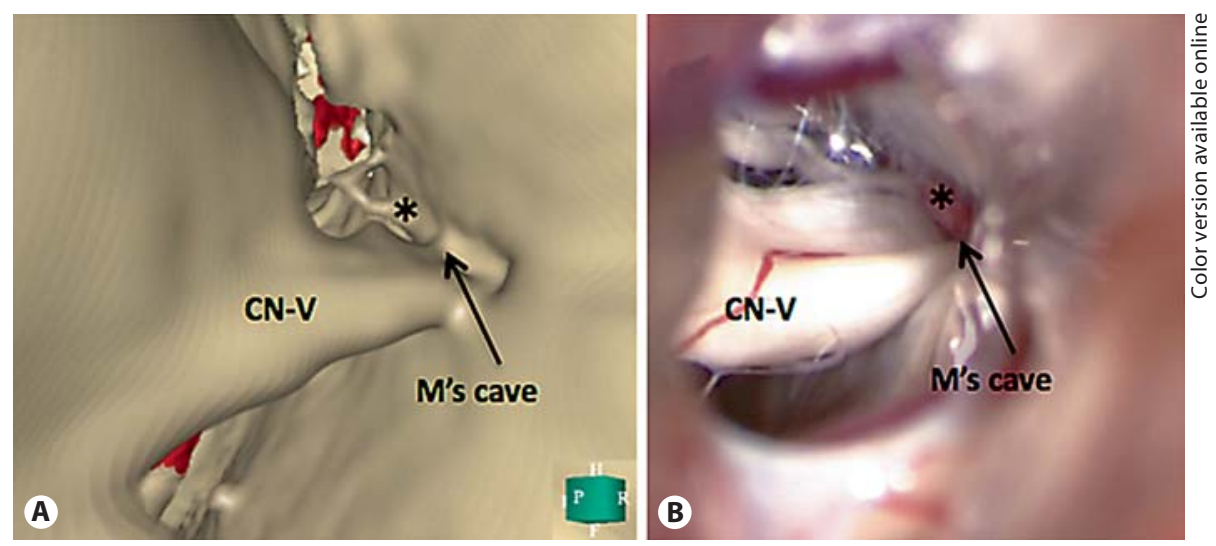

respond to a TPV, running medially to the trigeminal nerve and bridging into Meckel's cave. VE analysis of the 3D MFVI data imitating the surgical view via the retrosigmoid approach predicted that the compression site of the nerve at Meckel's cave could not be observed by surveying only from the usual surgical angle (fig. $3 \mathrm{~A}, \mathrm{E}$ ). No arterial compression was observed on the entire nerve route from the root exit zone to Meckel's cave in all 3 patients, and the SCA in cases 2 and 3 was confirmed to adhere to the tentorial surface.

\section{Intraoperative Findings and Surgical Outcomes}

We performed MVD via a retrosigmoid craniotomy. During the operation, no arterial compression was observed on careful viewing of the entire nerve route in the cistern in all cases, and the SCA previously adhered to the tentorium was also confirmed in cases 2 and 3. Anatomical information obtained from VE review on 3D MFVI was helpful to find the vein running behind the nerve in the surgical view. The vein bridged from the surface of the pons into the inside of Meckel's cave and compressed the superomedial site of the nerve inside or in front of the cave. We could find the vein only by retracting down the nerve, as to be completely comparable to preoperative 3D MFVI results (fig. 3). All those veins were coagulated and cut after dissection of the arachnoid around Meckel's cave.

Finally, all patients achieved a pain-free state without carbamazepine administration; however, patient 2 continued to experience a low-grade pain postoperatively, which disappeared gradually within 1 month. Partial facial numbness and hypesthesia were observed as postoperative complications in cases 1 and 3, but they recovered within 1 year in case 3 . Those patients accepted their surgical outcome as satisfactory.

Trigeminal Neuralgia Caused by

Transverse Pontine Vein
Draining and Bridging Patterns of the TPV in the Analysis of 50 Sides

In total, 50 bridging veins from the TPV were identified in the venous phase of 3D CTA in 45 of the 50 sides. The bridging and draining patterns identified by analysis on 3D MFVI were classified as shown in figure 4. A frequent bridging pattern was observed in $38 \%$ as bridging to the pyramidal wall inferiorly to Meckel's cave and then draining into the various venous sinuses (fig. 4A). The bridging pattern of passing near the trigeminal nerve was observed in $40 \%$, superiorly in $22 \%$ (types a and b) and inferiorly in $18 \%$ (type c), and connecting to the superior petrosal vein in $30 \%$ (types b and c; fig. 4B). The bridging pattern of draining medially to the nerve was observed in $14 \%$ (fig. 4C). Draining into Meckel's cave was found in only 4 (8\%) patients, including cases 2 and 3 (fig. 4D). Therefore, in this result, $50 \%$ of the bridging pattern was associated with TN.

\section{Discussion}

Although veins sometimes contribute to the NVC in combination with arteries in $\mathrm{TN}$, venous compression alone should be rare, almost $10 \%$ as listed in table $2[3,4$, 6-8]. The 3 cases presented in this study implied that the specific bridging pattern of the TPV could frequently associate with TN. Of course, we should note the fact that facial numbness after coagulating the vein appeared in case 1 and temporally in case 3 . Operative injury to the nerve may temporally alleviate the trigeminal pain to some extent. However, the anatomical and functional correlation in those patients should be persuasive for their pain. Pain restricted to only the V1 territory, as pre- 


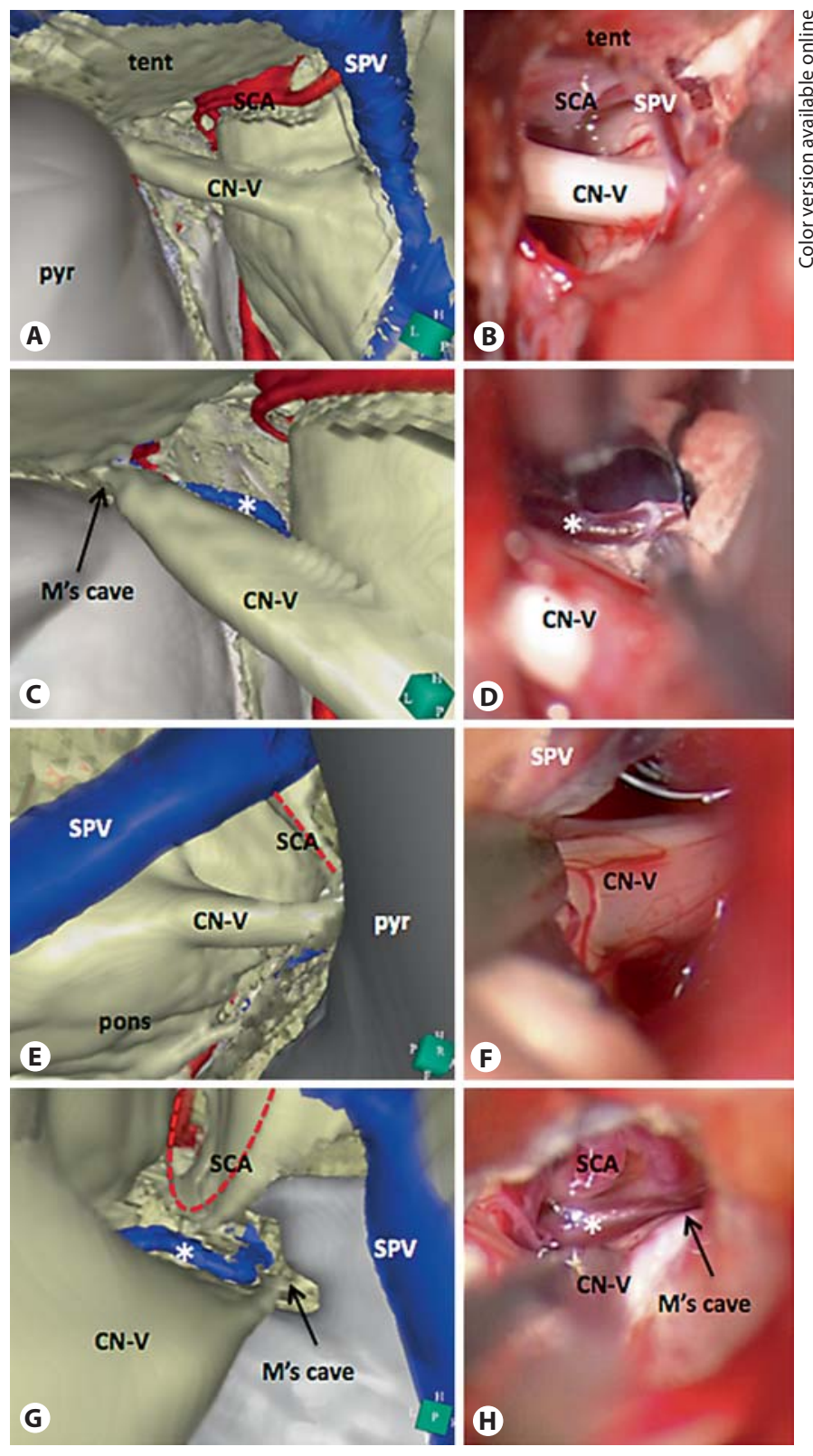

Fig. 3. Comparison between VE views on $3 \mathrm{D}$ MFVI data and surgical microscopic views in cases 2 (A-D) and 3 (E-H). Views on 3D MFVI (A, C, E, G) correspond to the microsurgical views $(\mathbf{B}, \mathbf{D}, \mathbf{F}, \mathbf{H})$, respectively. In both cases, the trigeminal nerve $(\mathrm{CN}$ $\mathrm{V})$ appears completely free from any vascular contact via a standard retrosigmoid approach $(\mathbf{A}, \mathbf{B}, \mathbf{E}, \mathbf{F})$. The viewing projection via the infratentorial supracerebellar route with retracting the trigeminal nerve can expose the vein, which is draining from TPVs (*), running into Meckel's (M's) cave behind the trigeminal nerve $(\mathbf{C}, \mathbf{D}, \mathbf{G}, \mathbf{H})$. SCAs are away from trigeminal nerves on 3D $\operatorname{MFVI}(\mathbf{A}, \mathbf{G})$ and still adhere to the tentorium on surgical views $(\mathbf{B}, \mathbf{H})$. pyr $=$ Pyramis; $\mathrm{SPV}=$ superior petrosal vein; tent $=$ tentorium.
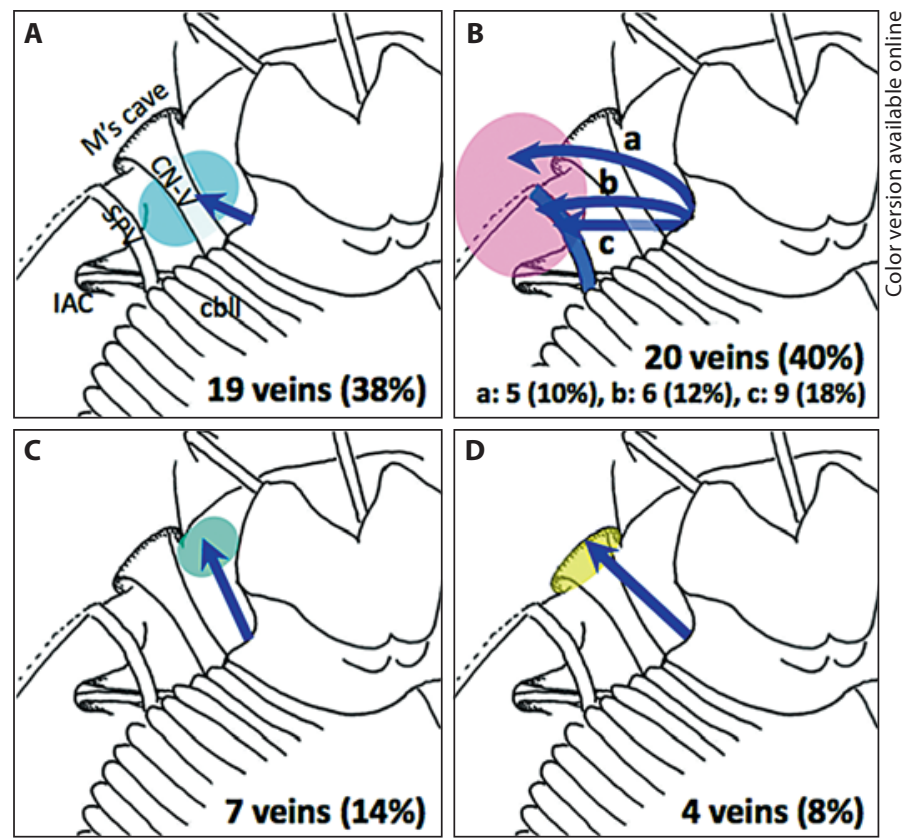

Fig. 4. Variation of the bridging pattern of the TPV. A The pattern bridging to the pyramidal wall inferiorly to Meckel's (M's) cave. cbll = Cerebellum; $\mathrm{CN}-\mathrm{V}=$ trigeminal nerve; $\mathrm{IAC}=$ internal auditory canal; SPV = superior petrosal vein. $\mathbf{B}$ The pattern bridging beyond the trigeminal nerve and laterally draining to Meckel's cave. This pattern is classified into 3 types draining into the tentorium (a), running over the trigeminal nerve to drain to the superior petrosal vein (b), and running under the trigeminal nerve to drain to the superior petrosal vein (c). C The pattern bridging directly to the inferior petrosal sinus or cavernous sinus medially to Meckel's cave. D The pattern bridging into Meckel's cave.

Table 2. Venous compression for $\mathrm{TN}$ in previous reports

\begin{tabular}{lrll}
\hline & $\begin{array}{l}\text { Patients } \\
\mathrm{n}\end{array}$ & $\begin{array}{l}\text { Participation of } \\
\text { vein in neurovas- } \\
\text { cular complex, \% }\end{array}$ & $\begin{array}{l}\text { Vein alone as } \\
\text { the offending } \\
\text { vessel, \% }\end{array}$ \\
\hline Barker et al. [3] & 1,204 & 68.2 & 12.6 \\
Lorenzoni et al. [4] & 82 & 13.4 & 9.8 \\
Hamlyn and King [6] & 41 & 22.0 & 9.8 \\
Kabatas et al. [7] & 62 & 51.6 & 17.7 \\
Matsushima et al. [8] & 121 & 20.7 & 5.8 \\
Sindou et al. [23, 24] & 350 & 24.7 & not clarified \\
Our series & 35 & 17.1 & 8.5 \\
\hline
\end{tabular}


sented by cases 1 and 2, is extremely rare, reported as 3\% in 1,185 patients [3]. Considering the functional anatomy of the trigeminal nerve [21], compression at the superomedial site of the nerve near Meckel's cave can directly affect the V1 route. A study in a large series of patients has already confirmed that the nerve was compressed anteriorly and superomedially to the root when pain was restricted to the V1 territory [22, 23]. To diagnose this type of neurovascular compression, the use of an advanced 3D MFVI technique was useful and reliable. Although numerous reports have suggested 3D imaging analysis based on MRI data for the evaluation of NVC [11-16], we present the first report of an attempt to use 3D MFVI with the integration of arterial and venous information obtained by MDCT. Presently, this analysis must be the best method to visualize the precise 3D anatomy consisting of cranial nerves and vasculatures in the cistern. The accuracy of predicted anatomies was finally confirmed in comparison with intraoperative findings and postoperative pain outcomes.

There have been a few studies focused on veins in relation to TN $[8-10,24]$, and only 1 study by Matsushima et al. [8] systematically investigated the venous compression as the cause of TN. They observed the participation of venous compression in 25 (20.7\%) of 121 surgical cases with $\mathrm{TN}$, but venous compression alone was observed in only 7 cases (5.8\%). Generally, the venous draining pattern around Meckel's cave is quite variable, balanced among a superior petrosal vein, a transverse pontine vein, the vein of the cerebellopontine fissure and a pontotrigeminal vein [25]. These veins bridge to the pyramidal and tentorial walls after merging together as the superior petrosal vein or bridge separately to the walls. The TPV was reported as the most frequent offending vessel of venous compression $[8,24]$. A previous cadaveric study explained that the vein usually attached to the superior or inferior surface of the trigeminal nerve just in front of Meckel's cave [25]. In our additional study on venous information in $50 \mathrm{CPA}$ regions with no tumor or vascular lesion, the varieties of the bridging patterns of TPV were successfully classified. The pattern bridging into Meckel's cave was rarely observed, in $8 \%$, but $50 \%$ with this pattern were the cause of $\mathrm{TN}$. When the vein drains into Meckel's cave, which is the space surrounded by dural and bony walls, compression to the nerve inside the cave may be inevitable. We found only 1 description about the vein possibly corresponding to this bridging pattern of the TPV as 'an aberrant trigeminal vein' [24]. We assume that the present type of venous compression may sometimes be missed in a presurgical evaluation, and such cas- es possibly fail to obtain pain relief after the usual MVD surgery, like in cases 2 and 3.

The use of the advanced 3D MFVI technique played an important role in diagnosis and surgical decision in the present cases. Our 3D MFVI featured the brain and nerves obtained from MRI-CISS, the skull from CT, and the vasculature from CTA obtained by MDCT, and the quality of imaging was sufficient to evaluate the shape of the trigeminal nerve and even of small vessels in the CPA region. Fundamentally, the main reason to apply the 3D imaging technique to the neurosurgical field is to clearly understand the complicated and unpredictable 3D brain anatomy before actual surgery, thereby executing the surgical procedure more safely and precisely. Especially in MVD surgery, precise knowledge of structural relations in the cistern of the CPA region is required because of numerous variations in the microsurgical anatomy of this region in individual patients. Several authors employed and developed the 3D imaging techniques in the presurgical evaluation for MVD [11-16]. Recently, the quality of imaging has improved to a greater extent than before $[12,16]$. The VE analysis has been reported as the method to obtain excellent visualization of anatomies in the cistern by reviewing freely from the viewpoint of a 'virtual camera' $[15,16]$. This technique allows us not only to predict the actual operative window from angles imitating surgical approaches, but also to observe the anatomies from angles impossible to obtain during surgery. For the diagnosis of TN, the evaluation of the entire nerve route from the root at the brainstem to Meckel's cave is necessary [26]. Therefore, in the present cases, VE analysis was helpful in exposing the vein hidden behind the trigeminal nerve.

To diagnose NVC, MRI has been used as routine radiological evaluation. Especially heavy $\mathrm{T}_{2}$-weighted imaging, such as 3D CISS, is well known to clearly visualize fine nerves and vessels with excellent contrast between structures in the cistern and the CSF [11, 27-30]. The combined use of MRA with 3D CISS has provided good sensitivity in the confirmation of arterial compression to the trigeminal nerve $[11,27,29]$. On the other hand, recent studies verified that the mere vascular contact with the trigeminal nerve was often observed in asymptomatic patients and was neither necessary nor sufficient for diagnosing $\mathrm{TN}[4,28,31,32]$. The finding of nerve deformity or distortion by vascular compression is believed to be more important and specific to the symptoms of TN $[4,6,28,31,32]$. In this respect, high-quality $3 \mathrm{D}$ imaging analysis provides intuitive evaluation of not only vascular contact, but also the form of the nerve itself. In the pres- 
ent 3 cases, depiction of the focal deformity of the trigeminal nerve at the compression site also supported the diagnosis of TN and the surgical decision.

We selected the retrosigmoid route for the surgical approach in all 3 patients. The TPV in the demonstrated patients bridged into the upper wall of Meckel's cave with the compression of the superomedial site of the trigeminal nerve. Meckel's cave is often hidden by a large bony bulging named the suprameatal process via a standard retrosigmoid approach [33]. In addition, 3D MFVI simulation in the present cases also predicted that the vein would be obscured by the trigeminal nerve itself. Therefore, we observed the cave and vein from a more superomedial viewpoint through the infratentorial supracerebellar route [33], as simulated on 3D MFVI. To accomplish MVD for the nerve, we sacrificed the bridging vein by coagulating and cutting it in all patients. In case of arterial compression, transpositioning of the offending artery to free the nerve from any contact is believed to be important to assure a satisfactory surgical outcome [5]. However, the presented pattern of vein was fixed in Meckel's cave and showed no mobility to free the nerve from vascular contact. There is no obvious consensus about the safety of cutting the draining veins in the CPA region [24, $25,34]$. We always preserve the main trunk of the superior petrosal vein bridging to the sinus. However, the TPV is just a part of the venous circulation connecting vertically and horizontally on the pontine surface [25], and also, the presented TPV bridged into Meckel's cave independently from the major draining system. Furthermore, the recurrence of TN caused by veins was reported at a high rate, and the most common cause of recurrence is also speculated to be the development of new veins [10]. We believe that the present pattern of the bridging vein from the TPV should be coagulated and cut completely, and cleaning out the arachnoid around the trigeminal nerve and Meckel's cave is also important to prevent the recurrence of $\mathrm{TN}$ in the patient. In coagulating the vein, the cause of the complications that cases 1 and temporally case 3 presented is thought to be the spread of electrical current heat for coagulation. We need to perform the procedure while paying special attention to avoid electrical injury to the nerve.

\section{Conclusion}

We reported 3 surgical cases with the specific bridging pattern of the TPV that could frequently be associated with TN, mainly in the V1 territory. The bridging vein runs behind the trigeminal nerve unnoticeable from a standard retrosigmoid approach and drains into Meckel's cave with compression at the superomedial site of the nerve. The 3D MFVI analysis with fusion of arterial and venous information from CTA data scanned by the advanced MDCT should be the best method to confidently expose the offending vein, which is prone to be missed, and to allow practical evaluation of the surgical anatomy.

\section{References}

1 Gardner WJ: Concerning the mechanism of trigeminal neuralgia and hemifacial spasm. J Neurosurg 1962;19:947-958.

$\checkmark 2$ Jannetta PJ: Arterial compression of the trigeminal nerve at the pons in patients with trigeminal neuralgia. J Neurosurg 1967;26: 159-162.

-3 Barker FG, Jannetta PJ, Bissonette DJ, Larkins MV, Jho HD: The long-term outcome of microvascular decompression for trigeminal neuralgia. N Engl J Med 1996;334:1077-1083.

-4 Lorenzoni JG, Massager N, David P, Devriendt D, Desmedt F, Brotchi J, Levivier M: Neurovascular compression anatomy and pain outcome in patients with classic trigeminal neuralgia treated by radiosurgery. Neurosurgery 2008;62:368-376.
5 Sindou M, Leston JM, Decullier E, Chapuis F: Microvascular decompression for trigeminal neuralgia: the importance of a noncompressive technique - Kaplan-Meyer analysis in a consecutive series of 330 patients. Neurosurgery 2008; 63(ONS suppl 2):ONS341351.

6 Hamlyn PJ, King TT: Neurovascular compression in trigeminal neuralgia: a clinical and anatomical study. J Neurosurg 1992;76: 948-954.

7 Kabatas S, Karasu A, Civelek E, Sabanci AP, Hepgul KT, Teng YD: Microvascular decompression as a surgical management for trigeminal neuralgia: long-term follow-up and review of the literature. Neurosurg Rev 2009;32:87-93.

8 Matsushima T, Huynh-Le P, Miyazono M: Trigeminal neuralgia caused by venous compression. Neurosurgery 2004;55:334-339.
9 Hebig GM, Callahan JD, Cohen-Gadol AA: Variant intraneural vein-trigeminal nerve relationships: an observation during microvascular decompression surgery for trigeminal neuralgia. Neurosurgery 2009;65:958961.

10 Lee SH, Levy EI, Scarrow AM, Kassam A, Jannetta PJ: Recurrent trigeminal neuralgia attributable to veins after microvascular decompression. Neurosurgery 2000;46:356361.

11 Akimoto H, Nagaoka T, Nariai T, Takada Y, Ohno K, Yoshino N: Preoperative evaluation of neurovascular compression in patients with trigeminal neuralgia by use of threedimensional reconstruction from two types of high-resolution magnetic resonance imaging. Neurosurgery 2002;51:956-961. 
-12 Kin T, Oyama H, Kamada K, Aoki S, Ohtomo $\mathrm{K}$, Saito N: Prediction of surgical view of neurovascular decompression using interactive computer graphics. Neurosurgery 2009; 65:121-129.

13 Lorenzoni J, David P, Devriendt D, Desmedt F, De Witte O, Massager N: Patterns of neurovascular compression in patients with classic trigeminal neuralgia: a high-resolution MRI-based study. Eur J Radiol 2009, Epub ahead of print.

- 14 Miller J, Acar F, Hamilton B, Burchiel K: Preoperative visualization of neurovascular anatomy in trigeminal neuralgia. J Neurosurg 2008;108:477-482.

15 Oishi M, Fukuda M, Takao T, Ishida G, Sato M, Fujii Y: The utility of presurgical simulation of microvascular decompression by MR virtual endoscopy. No Shinkei Geka (Jpn) 2007;35:1087-1095

16 Takao T, Oishi M, Fukuda M, Ishida G, Sato M, Fujii Y: Three-dimensional visualization of neurovascular compression: presurgical use of virtual endoscopy created from magnetic resonance imaging. Neurosurgery 2008;63(ONS suppl 1):ONS141-148.

$\checkmark 17$ Lell MM, Anders K, Uder M, Klotz E, Ditt H, Vega-Higuera F, Boskamp T, Bautz WA, Tomandl BF: New techniques in CT angiography. Radiographics 2006;26:S45-S62.

- 18 Matsumoto M, Kodama N, Sakuma J, Sato S, Oinuma M, Konno Y, Suzuki K, Sasaki T, Suzuki K, Katakura T, Shishido F: 3D-CT arteriography and 3D-CT venography: the separate demonstration of arterial-phase and venous-phase on 3D-CT angiography in a single procedure. AJNR Am J Neuroradiol 2005;26:635-641.
19 Oishi M, Fukuda M, Ishida G, Saito A, Hiraishi T, Fujii Y: Presurgical simulation using advanced 3D multifusion volumetric imaging in patients with skull-base tumors. Neurosurgery 2011;68:ONS188-ONS199.

20 Calhoun PS, Kuszyk BS, Heath DG, Carley JC, Fishman EK: Three-dimensional volume rendering of spiral CT data: theory and method. Radiographics 1999;19:745-764.

21 Gudmundsson K, Rhoton AL Jr, Rushton JG: Detailed anatomy of the intracranial portion of the trigeminal nerve. J Neurosurg 1971;35: 592-600.

22 Choudhari KA: Superior petrosal vein in trigeminal neuralgia. Br J Neurosurg 2007;21 288-292.

23 Sindou MP, Chiha M, Mertens P: Anatomical findings observed during microsurgical approaches of the cerebellopontine angle for vascular decompression in trigeminal neuralgia (350 cases). Stereotact Funct Neurosurg 1994;63:203-207.

24 Sindou M, Chiha M, Mertens P: Anatomical findings in microsurgical vascular decompression for trigeminal neuralgia: Correlations between topography of pain and site of the neuro-vascular conflict. Acta Neurochir 1995;64(suppl):125-127.

25 Matsushima T, Rhoton AL Jr, de Oliveira E, Peace D: Microsurgical anatomy of the veins of the posterior fossa. J Neurosurg 1983;59: 63-105.

26 McLaughlin MR, Jannetta PJ, Clyde BL, Subach BR, Comey CH, Resnick DK: Microvascular decompression of cranial nerves: lessons learned after 4,400 operations. J Neurosurg 1999;90:1-8.

-27 Anderson VC, Berryhill PC, Sandquist MA, Ciaverella DP, Nesbit GM, Burchiel KJ High-resolution 3-D magnetic resonance angiography and 3-D spoiled gradient-recalled imaging in the evaluation of neurovascular compression in patients with trigeminal neuralgia: a double-blind pilot study. Neurosurgery 2006;58:666-671.
28 Kakizawa Y, Seguchi T, Kodama K, Ogiwara T, Sasaki T, Goto T, Hongo K: Anatomical study of the trigeminal and facial cranial nerves with the aid of 3.0-tesla magnetic resonance imaging. J Neurosurg 2008;108:483490.

29 Yoshino N, Akimoto H, Yamada I, Nagaoka T, Tetsumura A, Kurabayashi T, Honda E, Nakamura S, Sasaki T: Trigeminal neuralgia: evaluation of neuralgic manifestation and site of neurovascular compression with 3D CISS MR imaging and MR angiography. Radiology 2003;228:539-545.

30 Yousry I, Camelio S, Schmid UD, Horsfield MA, Wiesmann M, Brückmann H, Yousry TA: Visualization of cranial nerves I-XII: value of $3 \mathrm{D}$ CISS and $\mathrm{T}_{2}$-weighted FSE sequences. Eur Radiol 2000;10:1061-1067.

>31 Miller JP, Acar F, Hamilton BE, Burchiel KJ: Radiographic evaluation of trigeminal neurovascular compression in patients with and without trigeminal neuralgia. J Neurosurg 2009;110:627-632.

\32 Peker S, Dinçer A, Necmettin Pamir M: Vascular compression of the trigeminal nerve is a frequent finding in asymptomatic individuals: 3-T MR imaging of 200 trigeminal nerves using 3D CISS sequences. Acta Neurochir 2009;151:1081-1088.

33 Hitotsumatsu T, Matsushima T, Inoue T: Microvascular decompression for treatment of trigeminal neuralgia, hemifacial spasm, and glossopharyngeal neuralgia: three surgical approach variations: technical note. Neurosurgery 2003;53:1436-1441.

-34 Masuoka J, Matsushima T, Hikita T, Inoue E: Cerebellar swelling after sacrifice of the superior petrosal vein during microvascular decompression for trigeminal neuralgia. J Clin Neurosci 2009;16:1342-1344. 